EPJ Web of Conferences 47, 03001 (2013)

DOI: $10.1051 /$ epjconf/20134703001

(C) Owned by the authors, published by EDP Sciences, 2013

\title{
TRAPPIST-UCDTS: A prototype search for habitable planets transiting ultra-cool stars
}

\author{
M. Gillon ${ }^{1, a}$, E. Jehin ${ }^{1}$, A. Fumel ${ }^{1}$, P. Magain ${ }^{1}$ and D. Queloz ${ }^{2}$ \\ ${ }^{1}$ Institut d'Astrophysique et de Géophysique, Université de Liège, Allée du 6 août 17, \\ Sart Tilman, Liège 1, Belgium \\ ${ }^{2}$ Observatoire de Genève, Université de Genève, 51 Chemin des Maillettes, 1290 Sauverny, \\ Switzerland
}

\begin{abstract}
The $\sim 1000$ nearest ultra-cool stars (spectral type M6 and latter) represent a unique opportunity for the search for life outside solar system. Due to their small luminosity, their habitable zone is 30-100 times closer than for the Sun, the corresponding orbital periods ranging from one to a few days. Thanks to this proximity, the transits of a habitable planet are much more probable and frequent than for an EarthSun analog, while their tiny size ( $\sim 1$ Jupiter radius) leads to transits deep enough for a ground-based detection, even for sub-Earth size planets. Furthermore, a habitable planet transiting one of these nearby ultra-cool star would be amenable for a thorough atmospheric characterization, including the detection of possible biosignatures, notably with the near-to-come JWST. Motivated by these reasons, we have set up the concept of a ground-based survey optimized for detecting planets of Earth-size and below transiting the nearest Southern ultra-cool stars. To assess thoroughly the actual potential of this future survey, we are currently conducting a prototype mini-survey using the TRAPPIST robotic $60 \mathrm{~cm}$ telescope located at $\mathrm{La}$ Silla ESO Observatory (Chile). We summarize here the preliminary results of this mini-survey that fully validate our concept.
\end{abstract}

\section{INTRODUCTION}

Confined for centuries to the rank of pure speculation, the existence of life outside our solar system is now at the edge of gaining its status of testable scientific hypothesis. Since the first discovery of a planet orbiting another sun-like star [1], more than 800 hundreds of such exoplanets have been detected at an ever increasing rate, not only gas and ice giants, but also a steeply growing fraction of terrestrial planets. In parallel, many projects aiming to characterize exoplanets have reached success, bringing notably first pieces of information on the atmospheric properties of giant exoplanets. Nearly all of these atmospheric studies have been made possible by the transiting configuration of the probed planets. Indeed, the special geometrical configuration of transiting planets offers the detailed study of their atmosphere without the cost of spatially resolving them from their host stars. The first atmospheric studies of transiting 'hot Jupiters' performed with space- and ground-based instruments have opened the era of comparative exoplanetology [2].

Exporting the techniques developed for these pioneering first studies of transiting gas giants to the atmospheric characterization of terrestrial planets seems to be a very promising path to search for life outside our solar system in the near future, using planned facilities like JWST or the E-ELT. The relevance of this approach relies on the discovery of suitable transiting planets, i.e. habitable terrestrial planets transiting a host star bright and small enough to lead to adequate signal-to-noise

\footnotetext{
ae-mail: michael.gillon@ulg.ac.be
}

This is an Open Access article distributed under the terms of the Creative Commons Attribution License 2.0, which permits unrestricted use, distribution, and reproduction in any medium, provided the original work is properly cited. 


\section{EPJ Web of Conferences}

ratios (SNRs) on spectroscopic biosignatures, assuming realistic observational programs with future facilities. Most studies in this domain have focused on JWST, e.g. [3][4], and they all agree that the best target for biosignatures detection with JWST would be an habitable terrestrial planet transiting one of the nearest ultra-cool stars (spectral type M6 or latter). Scaling the JWST SNRs presented in [4] for Earth-size planets, and basing on the densities of ultra-cool stars in the close solar neighborhood [5], we derive a total number of $\sim 1000$ nearby ultra-cool stars for which a transiting Earth-twin could have its atmospheric properties thoroughly studied with JWST. Because of the proximity of their habitable zone, this relatively small number of targets is balanced by their favorable geometric transit probability. Even if only $10 \%$ of them has a habitable planet, there should be at least 2 to 3 of these planets waiting to be caught in transit and studied by JWST and other future facilities.

On paper, a dedicated ground-based transit survey operating from one or several world class observatories with modest-size telescopes (up to $1 \mathrm{~m}$ ) and near-IR-optimized CCD detectors should be able to probe efficiently (Earth-size and below) the habitable zone of these $\sim 1000$ nearby ultracool stars within a few years. Indeed, the small size of ultra-cool stars leads for Earth-size planets to transit depths from a few mmags up to $>1 \%$, similar to the typical transit depth of a "hot Jupiter". Furthermore, the proximity of the habitable zone translates into orbital periods of only a few days, leading to needed photometric monitoring of much smaller duration than for bona fide Earth-Sun twin systems. A particularity of such survey is that each target would have to be monitored individually and continuously, as nearby ultra-cool stars are spread all over the sky and as the highest possible precision and cadence would be required to efficiently probe the terrestrial regime.

While promising on paper, the actual potential of such a survey concept has to be empirically demonstrated. In addition to their intrinsic faintness, latest M-dwarfs are commonly considered as active objects. This activity could be a big issue, as it could a priori strongly limit the ability to detect low-amplitude transits. Another possible barrier could come from the atmosphere itself. Indeed, in the very-near-IR, the water molecule and $\mathrm{OH}$ radical contributes a number of absorption bands (as well as significant emission for $\mathrm{OH}=$ airglow) which bring unavoidable important levels of red noise in photometric time-series. For these reasons, a thorough assessment study based on a prototype survey was mandatory to validate our concept.

\section{TRAPPIST/UCDTS}

In 2011, we initiated a mini-survey using the $60 \mathrm{~cm}$ robotic telescope TRAPPIST (TRAnsiting Planets and PlanetesImals Small Telescope; [6]) located at ESO La Silla Observatory (Chile) to monitor 50 among the nearest and brightest Southern ultra-cool stars. TRAPPIST is equipped with a near-IR optimized CCD camera offering excellent quantum efficiencies from 300 to >900nm, making it a good prototype for the envisioned survey. This mini-survey is called UCDTS (Ultra-Cool Dwarfs Transit Survey), and its concept is to monitor in a wide near-IR filter each ultra-cool star during at least 50 hours spread over several nights, with the goals to assess the typical photometric precisions that can be reached for these stars on nightly timescales, the resulting detection thresholds for terrestrial planets, and to identify in the mean time the astrophysical and atmospheric limitations of the concept.

So far, 20 UCS have been monitored, including 5 M6, 6 M7, 4 M8 and 5 M9. The main results are as follows:

- 12 out of the 20 observed targets show stable photometry on nightly timescale. Five of the others show clear flares in some light curves, all 5 having M6 or M7 spectral type (Fig. 1). These flares are seen in our near-IR light curves as sudden increase of a few percents of the measured brightness, followed by a gradual decrease back or close to the normal level, the whole process taking only 10 to $30 \mathrm{~min}$. In the context of a transit search, it is easy to identify and discard the affected portions of light curves. Furthermore, their frequency is small (1 flares per 3-4 nights in average). For three other targets, there is a periodic modulation of the measured fluxes on a timescale of 1.5 to 4 hours which could correspond to the rotation period of the star (Fig. 1). These modulations are easily modeled 


\section{Hot Planets and Cool Stars}
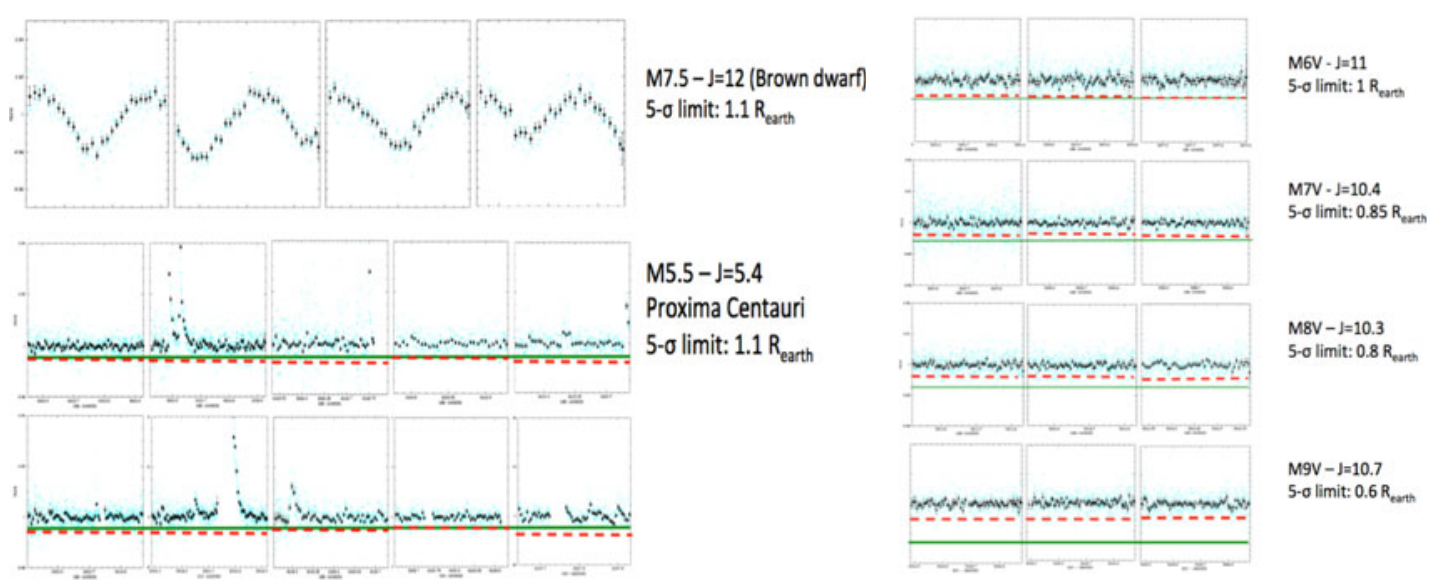

Figure 1. Le ft : TRAPPIST/UCDTS light curves for the two most variable stars of our observed sample. For each light curve, the measurements are shown unbinned and binned per $0.005 \mathrm{~d}(7.2 \mathrm{~min})$. Top: the photometry of this M7.5 object illustrates the photometric modulation of a fast rotation ultra-cool star (here, it is in fact a young brown dwarf). The derived rotation period is $2.8 \mathrm{~h}$. Bottom: 10 nights of photometry for our closest neighbor, Proxima Centauri. This star is the most active one among our sample so far. The dashed red lines below the light curves show the 5- $\sigma$ transit detection threshold for a habitable planet derived from the injection of fake transits and odds ratio estimation through Bayesian model comparison. In these analyses, the data obtained during a flare were discarded. The green lines show the transit depth expected for an Earth-size planet. Right: typical TRAPPIST/UCDTS light curves obtained, from top to bottom, for a M6, M7, M8 and M9 star. These light curves were all detrended by a second-order time polynomial to correct for differential extinction and smooth stellar variability. The 5- $\sigma$ transit detection thresholds (red) and the expected transit depth for an Earth-size planet (green) are shown as for Proxima Cen.
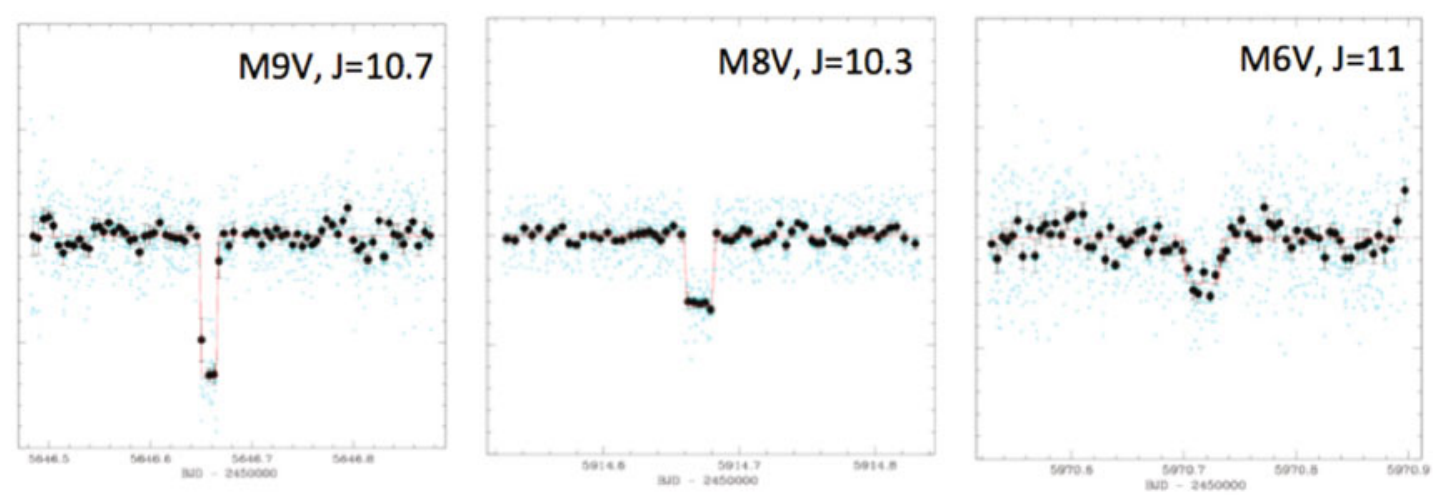

Figure 2. Actual TRAPPIST/UCDTS light curves for three ultra-cool stars, after injection of a fake transit of an habitable Earth-size planet. The best-fit transit models are over-imposed in red.

with simple sinus terms(s), and their typical frequencies are very different from the ones of a transit of a habitable Earth-like planet (short duration, very sharp ingress + egress).

- The reached photometric precisions are globally nominals. There is no hint of extra-amount of correlated noise except for a few nights with large humidity. Our data have allowed us to deduce an empirical calibration of the noise budget for ground-based near-IR CCD photometry of ultra-cool 
stars from La Silla, confirming that nominal sub-mmag precisions can be reached for these stars from a suitable astronomical site (good transparency, low humidity).

- Injecting fake single transits into the data, we have performed detailed Bayesian model comparisons on the light curves with and without transits, taking into account the correlation of the noise. Figure 2 shows some of our light curves after injection of a fake transit of Earth-size planet. We derived for these transits detection significances of 12, 7, and 4-sigma. Assuming a 5-sigma detection threshold, the average detection limits in terms of planetary radius are $1.08 R_{\oplus}$ for the M6 observed so far, 0.96 $R_{\oplus}$ for the M7, $0.93 R_{\oplus}$ for the M8, and $0.85 R_{\oplus}$ for the M9. It can be noticed in Fig. 2 that even at 4-sigma, the transit is still noticeable by eye for the M6, so these limits are conservative.

\section{PRELIMINARY CONCLUSION}

Our TRAPPIST-UCDTS mini-survey is not completed yet, but the results acquired so far are very promising. First, from intense simulations based on the injection and recovery of fake transits of terrestrial planets in our actual photometry, we have reached the conclusion that the variability of a fraction of ultra-cool stars (flares + rotation) does not limit the ability to detect transits of close-in planets. Second, our detection thresholds deduced from our data show that we are sensitive to transits of planets of Earth-size and below. Detecting effectively these planets requires now to go one step further and to observe a larger sample of nearby ultra-cool stars with a fully dedicated and optimized project.

\section{References}

[1] Mayor M. \& Queloz D., Nature, 378, 355-359 (1995)

[2] Seager S. \& Deming D., ARA\&A, 48, 631-672 (2010)

[3] Seager S., Deming D., \& Valenti J. A., Astrophysics in the Next Decade, Astrophysics and Space Science Proceedings (Springer, Netherlands, 2009), 123

[4] Kaltenegger L. \& Traub W A., ApJ, 698, 519-527 (2009)

[5] Reid I. N., Cruz K. L. Kirkpatrick J. D., et al., AJ, 136, 1290-1311 (2008)

[6] Gillon M., Jehin E., Magain P., et al., EPJ Web of Conference, 11, 06002 (2011) 